\title{
A percentagem na aprendizagem com compreensão dos números racionais
}

\section{Percentage in rational number learning with understanding}

\author{
Helena Gil Guerreiro ${ }^{1}$ \\ Lurdes Serrazina ${ }^{2}$ \\ João Pedro da Ponte ${ }^{3}$
}

\begin{abstract}
Resumo
Neste artigo discutimos a construção do conhecimento conceptual dos números racionais, por alunos do $3 .^{\circ}$ e $4 .^{\circ}$ ano do ensino básico, como processo integrado em que se privilegia a percentagem. Pretendemos perceber, numa etapa inicial da aprendizagem dos números racionais, que compreensão constroem os alunos da natureza relacional da percentagem e de que modo a percentagem contribui para essa aprendizagem, considerando uma compreensão das relações entre as diferentes representações os números racionais. Este estudo tem por base uma experiência de ensino, seguindo uma metodologia de Investigação Baseada em Design. Os dados foram recolhidos através da observação participante, apoiada num diário de bordo, e de gravações áudio e vídeo das aulas, sendo analisados os diálogos e as produções dos alunos de uma turma. Os resultados evidenciam que os alunos mostram compreender a natureza relacional da percentagem e que a compreensão das relações e conceitos envolvidos na noção de percentagem contribuem para a construção de um entendimento da natureza multiplicativa dos números racionais.
\end{abstract}

Palavras-chave: Aprendizagem; Números racionais; Conhecimento conceptual; Representações; Percentagem.

\section{Abstract}

In this article we discuss the construction of conceptual knowledge of rational numbers by basic education students in grades 3 and 4 as an integrated process with a focus on percentage. At an early stage of learning rational numbers, we aim to know what understanding students develop of the relational nature of percentage and how percentage contributes to this learning, considering an understanding of the relations among different rational number representations. This study is based on a teaching-learning experience, following a design based research methodology. Data was collected through participant observation, supported in a logbook, with audio and video recordings of the lessons and we analyze the dialogues and the productions of the students of a class. The results show that the students display an understanding of the relational nature of percentage and that the grasp of relationships and concepts involved in this notion contributes to building an understanding of the multiplicative nature of rational numbers.

\section{Submetido em: 19/12/2017 - Aceito em: 08/05/2018 - Publicado em: 13/06/2018}

${ }^{1}$ Doutoranda em Educação Matemática no Instituto de Educação, Universidade de Lisboa, Portugal. Professora no Agrupamento de Escolas Braamcamp Freire, Lisboa. Membro colaborador da Unidade de Investigação e Desenvolvimento em Educação e Formação (UIDEF), Instituto de Educação, Universidade de Lisboa, Portugal. Email: hg@campus.ul.pt.

${ }^{2}$ Doutora em Educação Matemática pela Universidade de Londres (UK). Professora Coordenadora Aposentada da Escola Superior de Educação, Instituto Politécnico de Lisboa, Lisboa, Portugal. Membro integrado da Unidade de Investigação e Desenvolvimento em Educação e Formação (UIDEF), Instituto de Educação, Universidade de Lisboa, Portugal. Email: lurdess@eselx.ipl.pt.

${ }^{3}$ Doutor em Educação Matemática pela Universidade da Georgia (UGA, EUA ). Professor Catedrático do Instituto de Educação, Universidade de Lisboa (IEUL), Lisboa, Portugal. Email: jpponte@ie.ulisboa.pt. 
Keywords: Learning; Rational numbers; Conceptual learning; Representations; Percentage

\section{Introdução}

A compreensão dos números racionais ${ }^{4}$ tem sido amplamente estudada, quer pela importância que assumem como alicerce de aprendizagens matemáticas futuras, quer pelas dificuldades que geram (Behr, Lesh, Post, \& Silver, 1983; Lamon, 2007). Contudo, a investigação neste tema continua a ser pertinente, dado o facto de serem poucos os progressos alcançados para um ensino-aprendizagem efetivo (Tian \& Siegler, 2017). Sendo importante saber que representação dos números racionais privilegiar na sua aprendizagem, são poucos os estudos recentes que se focam na compreensão da percentagem (Tian \& Siegler, 2017). Em particular, importa conhecer melhor o contributo que pode dar numa etapa inicial da compreensão dos números racionais.

Tradicionalmente, o ensino dos números racionais envolve um trabalho com frações, numerais decimais e percentagens de forma isolada, como tópicos distintos, sendo que todos eles oferecem dificuldades aos alunos (Moss, 2002; Tian \& Siegler, 2017). Alcançar uma compreensão aprofundada destas representações e das suas relações é crucial para trabalhar de forma flexível com os números racionais, como destaca o NCTM (2000), sendo este um desafio que a escola enrenta.

Numa perspetiva sociocultural, que considera que a aprendizagem se constrói na participação em interação social (Gravemeijer \& Cobb, 2006), desenvolvemos uma investigação que privilegia a percentagem numa etapa inicial da aprendizagem dos números racionais. Tomamos como ponto de partida o facto da percentagem, enquanto conceito matemático, permitir relacionar a vida real com a descoberta das estruturas multiplicativas, potenciando uma construção gradual da aprendizagem das diferentes representações simbólicas do número racional e das suas relações (Moss \& Case, 1999; Parker \& Leinhardt, 1995). Neste artigo temos como objetivos: 1) perceber que compreensão constroem os alunos da natureza relacional da percentagem numa etapa inicial da aprendizagem dos números racionais e 2) de que modo a perfcentagem contribui para a aprendizagem dos números racionais, numa etapa inicial, considerando uma compreensão das relações entre as diferentes representações destes números.

\section{Enquadramento teórico}

Compreensão dos números racionais como processo integrado de enriquecimento conceptual

As mudanças no ensino da matemática envolvem um esforço cada vez maior para tornar as experiências de aprendizagem mais cooperativas, mais conceptuais e mais interligadas (Dreyfus, 1999). O NCTM (2000) defende que os alunos devem aprender Matemática com compreensão, construindo ativamente os novos conhecimentos apoiados na

\footnotetext{
${ }^{4} \mathrm{O}$ termo números racionais remete para o conjunto dos números racionais não negativos. No currículo português estes aparecem após o trabalho com os números naturais com o zero.
} 
sua experiência e conhecimentos prévios. Esta ideia valoriza o conhecimento da vida real, aplicado e circunstancial, correto ou incorreto e muitas vezes não resulta diretamente de um ensino formal - conhecimento que Leinhardt (1988) designa por "intuitivo". Reforça que o ensino deve articular os novos conhecimentos com o que os alunos já sabem. Isto é, deve tomar como ponto de partida o conhecimento que os alunos possuem, intuitivo ou formal, e construir uma rede de relações com novos factos e informações, o que Hiebert e Lefevre (1986) designam como conhecimento "conceptual".

Esta perspetiva permite entender a compreensão dos números racionais como extensão dos conhecimentos numéricos que os alunos já possuem, quer intuitivos, quer conhecimentos sobre os números inteiros ${ }^{5}$. Leinhardt (1988) refere que "os números racionais representam a primeira extensão dos números para além dos números naturais” (p. 120). Esta extensão não muda o objeto em si, mas enriquece a conceção que os alunos têm acerca dos números, pois sobre ele vão aprender novos factos (Siegler, 2016). Ao contrário dos números inteiros, que são caracterizados pela correspondência um (símbolo) para um (grandeza) no sistema de numeração decimal, os números racionais podem ser representados de diversas formas, nomeadamente através de percentagens, frações ou numerais decimais. Estas representações, embora possuam notação diferente, traduzem, na sua essência, um mesmo número, a mesma grandeza numérica.

A teoria integrada do desenvolvimento do conceito de número destaca o caracter unificador da grandeza do número para a aprendizagem dos diferentes conjuntos numéricos (Siegler, Thompson, \& Schneider, 2011). Segundo esta teoria, é possível pensar a construção da compreensão dos números racionais com base na ideia de que estes números (inteiros e não inteiros), têm uma grandeza que pode ser representada e ordenada numa reta numérica, através de uma dada notação simbólica. Esta ideia apoia-se na hipótese de que os alunos representam a grandeza de um número simbolicamente numa reta numérica mental, como estrutura dinâmica que é primeiro usada para representar números inteiros pequenos e que, progressivamente estendem e ampliam, sendo subdividida para incluir outros números racionais (Siegler, 2016). Deste modo, o desenvolvimento numérico dos alunos, à medida que vão contactando e ganhando experiência com cada conjunto numérico, pode ser consubstanciado na finalidade de "criar representações da grandeza dos números cada vez mais precisas para um conjunto cada vez mais abrangente de números" (Siegler, 2016, p. 342).

No início deste processo de alargamento dos conhecimentos dos números inteiros aos números racionais Siegler, Fazio, Bailey e Zhou (2013) destacam que os alunos assumem a ideia de que as propriedades dos números inteiros são propriedades de todos os números. Importa assim que os alunos gradualmente possam ir integrando o conhecimento dos números fracionários no conhecimento relativo aos números inteiros que já possuem, num processo de enriquecimento conceptual contínuo (Siegler, Thompson \& Schneider, 2011).

\footnotetext{
${ }^{5} \mathrm{O}$ termo números inteiros remete para o conjunto dos números naturais com o zero.
} 


\section{A Percentagem como Representação dos Números Racionais}

Numa abordagem integrada do processo de desenvolvimento numérico, apoiada na propriedade da grandeza dos números, a construção do conhecimento dos números racionais resulta da interligação de redes de nós de conhecimentos parciais, num processo de mudança e reorganização, à medida que os alunos procuram coordenar e ligar pedaços de informação (Hiebert, Wearne, \& Taber, 1991). Assim, dado que a construção do conhecimento não decorre de forma linear, o processo de desenvolvimento numérico beneficia de uma abordagem contínua com diferentes sistemas de representação (NCTM, 2000). Com efeito, é a capacidade de fazer transformações, não só dentro de um sistema de representação, mas também entre sistemas, que permite ir construindo as relações entre os conceitos e gradualmente, dar significado aos conhecimentos relativos aos números racionais (Behr, Lesh, \& Post, 1981).

Frações, numerais decimais e percentagens são três representações dos números racionais. Umas são mais usadas que outras, em função do contexto, contudo, todas expressam os seus múltiplos significados (Tian \& Siegler, 2017). Goldin e Kaput (1996) referem que o poder de uma representação é tanto maior quanto maior for a sua versatilidade, isto é, a facilidade em ser aplicada a diferentes contextos e ser de uso eficiente, permitindo que se relacione e seja convertida noutra facilmente. Nesta perspetiva, a percentagem pode ser definida como uma representação versátil, caraterizada essencialmente por três aspetos. Em primeiro lugar, está presente na linguagem do quotidiano e a sua utilização está generalizada aos mais diversos contextos da sociedade, sendo intuitivo o entendimento da expressão "por cento", como por cada cem ou em cada cem, isto é, algo que se compara com cem. Em segundo lugar, a sua notação, na forma simples, como 10\%, envolve um numeral, com propriedades de número inteiro, e um símbolo, que lhe imprime uma relação multiplicativa. Esta notação apresenta-se mais próxima da notação dos números inteiros do que a fração ou o numeral decimal, em que dois números inteiros são usados, separados por um traço de fração ou por uma vírgula. Por último, a percentagem relaciona-se facilmente com as outras representações dos números racionais, na medida em que é simples converter qualquer percentagem em fração ou em numeral decimal.

A discussão sobre que representação deve ser privilegiada para iniciar o trabalho com os números racionais assume na investigação uma dicotomia entre o numeral decimal e em fração, estando a percentagem praticamente excluída (Tian \& Siegler, 2017). Porém, Moss e Case (1999), dando enfase à natureza multiplicativa dos números racionais, sugerem que a percentagem seja privilegiada numa fase inicial do trabalho com os números racionais, incidindo na sua dimensão relacional. Esta opção, que assumimos neste estudo, surge apoiada nos conhecimentos intuitivos que os alunos possuem em relação à proporção e nos conhecimentos que já têm dos números inteiros de 1 a 100 e que constituem a base para uma entrada no campo das relações multiplicativas (Moss, 2002; Moss \& Case, 1999). Estes autores descrevem uma abordagem aos números racionais através da percentagem que permite aos alunos ir integrando os seus conhecimentos informais de percentagem com 
estratégias de composição e decomposição dos números inteiros (Moss \& Case, 1999). Estas estratégias baseiam-se em diversas representações, ativas, icónicas e simbólicas (Bruner, 1996) e na linguagem oral e escrita (Ponte \& Serrazina, 2000). Paralelamente ao trabalho com a percentagem, os alunos são desafiados a fazer conversões preliminares com representações simbólicas de referência simples que já conhecem, percecionando gradualmente como se relacionam e iniciando o processo de movimentação entre elas.

Tal como referem Parker e Leinhardt (1995), apesar da aparente simplicidade da percentagem, trata-se de um conceito de aprendizagem complexa, muitas vezes gerador de conflitos. Isso é visível desde logo pela própria palavra que, em português corrente, encerra diferentes significados, podendo de acordo com o contexto, ser interpretada como uma taxa, a quantia que se obtém quando se aplica a taxa, uma informação estatística, um processo de cálculo ou um conceito matemático. Esta multiplicidade de significados mostra o quanto está presente no dia-a-dia, o que reforça a pertinência de antecipar a sua aprendizagem. No entanto, dada a sua complexidade, importa salvaguardar que o estudo da percentagem se foca na compreensão da sua natureza relacional e não apenas nas características da sua representação (Parker, 2004; Tian \& Siegler, 2017).

\section{Construção de um Conhecimento Conceptual da Percentagem}

Usualmente, a percentagem é trabalhada depois da fração e do numeral decimal e, embora envolva o uso de diversas representações, estas são rapidamente abandonadas e é dada enfase a conversões, equações e cálculos, que nem sempre destacam a natureza do conceito (Parker, 2004; Parker \& Leinhardt, 1995). Parker e Leinhardt (1995) afirmam ser importante ter em conta, desde logo, que a percentagem "é uma linguagem de proporção privilegiada que simplifica e condensa descrições de comparações multiplicativas" (p. 472). Isto é, trata-se de uma linguagem que na sua essência descreve relações que têm por base a proporcionalidade, mas que ficam escamoteadas pela sua notação, pelo que importa explicitar.

Embora o ensino da percentagem tenha em vista a proficiência dos alunos em procedimentos de cálculo, as ideias-chave subjacentes à construção do conceito de percentagem envolvem a compreensão da sua dimensão relacional (Parker \& Leinhardt, 1995). Estas ideias têm subjacente conhecimentos conceptuais que os alunos devem poder descobrir e relacionar ao longo da sua escolaridade e nos quais devem ancorar o desenvolvimento de um conhecimento procedimental flexível e eficaz (De Corte, Depaepe, Eynde, \& Verschaffel, 2005; Lembke \& Reys, 1994). Alguns desses aspetos conceptuais podem ser trabalhados no início da aprendizagem dos números racionais, altura em que os alunos mostram propensão para avaliar situações em termos multiplicativos e começam a entender a grandeza relativa dos números numa perspetiva de relações multiplicativas (Moss \& Case, 1999). Nomeadamente, os alunos precisam de compreender que a percentagem: 1) apesar da sua linguagem aparentemente aditiva (por exemplo, 10\% menos em relação ao valor inicial) traduz uma comparação entre dois números ou quantidades, de natureza multiplicativa; 2) possuí propriedades de número racional, pelo que não pode ser interpretada 
sem ter em conta o referente, e pode ser transformada numa fração ou num decimal; 3) traduz uma relação parte-todo, mas também de razão, envolvendo conjuntos diferentes; e 4) descreve uma situação fixa, representando como diferentes quantidades se relacionam entre si (De Corte et al., 2005; Parker \& Leinhardt, 1995; Van den Heuvel-Panhuizen, 2003).

A compreensão da percentagem não começa apenas quando o termo surge pela primeira vez na escola. Desde cedo que as crianças têm tendência para assumir que quantidades relativas mudam juntas, mostrando uma certa intuição na compreensão de situações de covariância simples, como refere Lamon (2007), o que apoia a propensão intuitiva dos alunos para a proporção (Moss \& Case, 1999). O contacto com a percentagem começa nas diversas situações da vida familiar, constituindo-se como informação com significado para os alunos. Esta ligação ao quotidiano fornece contextos reais, familiares aos alunos, que importa trazer para a sala de aula e reforça a importância de relacionar a aprendizagem formal da percentagem com os conhecimentos informais dos alunos (De Corte et al., 2005; Van den Heuvel-Panhuizen, 2003). Esta abordagem à percentagem encontra eco na Educação Matemática Realista (RME), que defende que "os alunos devem aprender matemática desenvolvendo ferramentas e conceitos matemáticos e a partir de situações problemáticas do dia-a-dia, que lhes façam sentido" (Van den Heuvel-Panhuizen, 2003, p. 9).

A experiência do dia-a-dia, com diferentes representações associadas à percentagem, como os ícones da bateria de um telemóvel ou a barra de estado de um download, permite que estas representações possam ser usadas como modelos na aprendizagem da percentagem, através de um processo de matematização progressiva da realidade (Freudenthal, 1968; Van den Heuvel-Panhuizen, 2003). Van Galen e Van Eerde (2013) referem a existência de várias representações, familiares aos alunos, e que constituem modelos para trabalhar a percentagem, como a barra da percentagem, a tabela de percentagem ou a reta numérica dupla. As escalas de comparação são outra representação que, permitindo interpretar com facilidade as relações inerentes à percentagem, podem ser convocadas para o trabalho com os alunos mais novos (Parker \& Leinhardt, 1995).

Van den Heuvel-Panhuizen (2003) descreve uma trajetória de aprendizagem da percentagem, em que a barra é usada como modelo de uma situação concreta para dar sentido à percentagem e relacionar representações dos números racionais. À medida que evoluem as relações numéricas que os alunos são capazes de estabelecer, a própria barra pode evoluir para um modelo mais independente dos contextos (Van Galen, Feijs, Figueiredo, Gravemeijer, Herpen, \& Keijzer, 2008). Com efeito, a barra pode assumir uma forma mais abstrata como reta numérica dupla, traduzindo a relação entre quantidades de duas grandezas distintas que variam em conjunto, constituindo um modelo a ser aplicado em diversos contextos (Van den Heuvel-Panhuizen, 2003).

Desta forma, as representações no trabalho com a percentagem constituem-se como modelos poderosos para promover a construção de um conhecimento conceptual, se derivam de contextos realistas e se são suficientemente flexíveis para serem usados em níveis mais avançados (De Corte et al., 2005). Esta atividade de modelação implica uma evolução do 
próprio modelo, "de modelos de situações concretas para modelos de pensamento" (Van Galen et al., 2008, p. 18) pelo que o papel dos diferentes sistemas de representação é "crucial na progressão do conhecimento informal para o formal, bem como a importância atribuída aos contextos" (Serrazina, 2012, p. 25).

Considerando estas dimensões da aprendizagem da percentagem, a compreensão que os alunos mostram da sua natureza relacional, destacando a comparação que permite fazer de algo com 100, enquanto proporção privilegiada, pode ser interpretada, numa etapa inicial, considerando vários aspetos. Lembke e Reys (1994) destacam a capacidade de: 1) interpretar a percentagem tendo como suporte uma representação icónica; 2) relacionar frações, decimais e percentagens; 3) mobilizar números de referência ou outras estratégias para estimar percentagens; 4) resolver mentalmente cálculos de percentagem na resolução de problemas; e 5) apreciar a razoabilidade da sua solução. Se a aprendizagem da percentagem começar pela compreensão da sua natureza relacional, os alunos percecionam as relações e comparações implícitas que oferece, centrando-se no que a percentagem efetivamente é (Parker \& Leinhardt, 1995) e, ao longo da sua escolaridade, terão oportunidade de se tornarem eficientes no seu cálculo.

\section{Metodologia}

Este estudo segue os procedimentos metodológicos de uma investigação baseada em design na sala de aula (Cobb et al., 2001; Ponte, Carvalho, Mata-Pereira, \& Quaresma, 2016). A modalidade escolhida apoiou-se na construção e implementação de uma experiência de ensino em sala de aula (Gravemeijer \& Cobb, 2006). O seu objetivo foi compreender o que os alunos aprendem e como aprendem no ambiente natural de aprendizagem, determinando que a recolha de dados fosse feita na sala de aula e que a escolha da unidade de análise recaísse sobre a própria turma. Envolveu, por um lado, uma dimensão de conteúdo matemático, que se inspirou no currículo experimental de Moss e Case (1999). Esta dimensão centrou-se num trabalho em torno das diferentes representações do número racional e das suas relações, traduzindo-se numa trajetória de aprendizagem prevista que se estruturou em três etapas (Figura 1). 


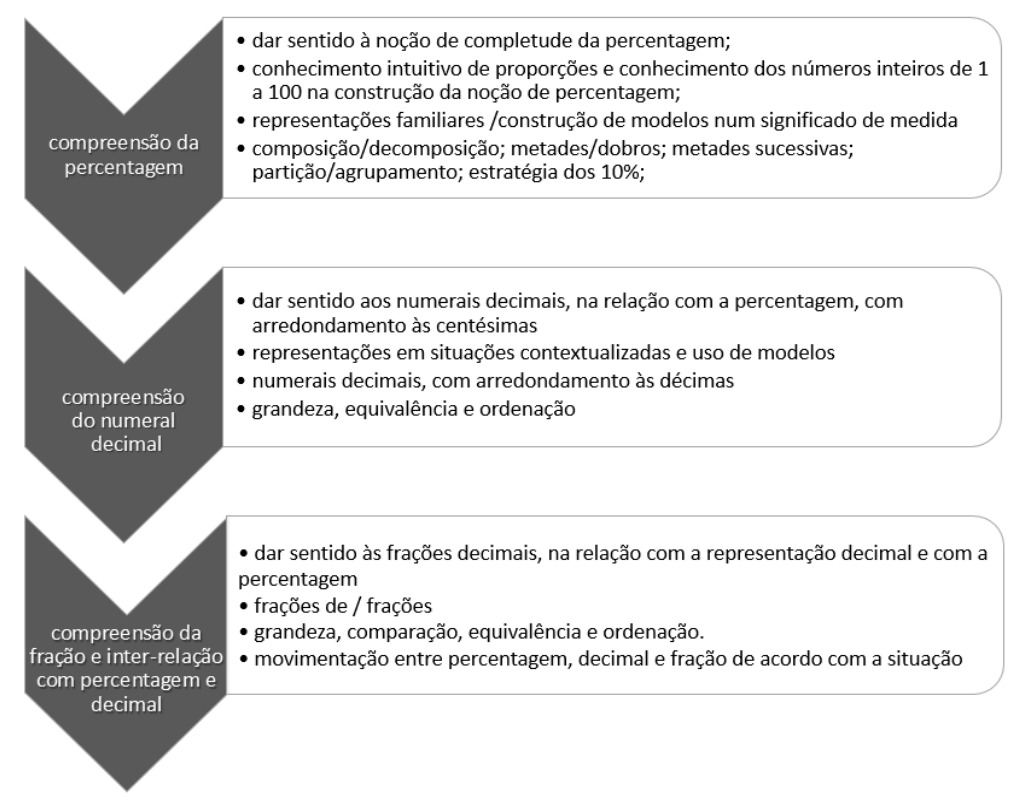

Figura 1 - Trajetória de aprendizagem prevista.

Fonte: Elaborado pelos autores.

Inicialmente focou-se na compreensão da percentagem, num significado de medida, a que se seguiu um enfoque na compreensão da representação decimal e, posteriormente, um trabalho em torno da compreensão da fração. Esta organização, embora não deva ser entendida como linear, conduziu a que a experiência de ensino se estruturasse em três microciclos, numa relação entre as ideias teóricas e o desenrolar dos acontecimentos na prática. Por outro lado, teve subjacente uma dimensão pedagógica, que procurou compreender os meios através dos quais a aprendizagem comparticipada pela turma aconteceu, no contexto social da sala de aula, num processo de ensino-aprendizagem de natureza exploratória (Ponte, 2005).

A experiência de ensino decorreu ao longo de dois períodos letivos, do $3 .^{\circ}$ e $4 .^{\circ}$ ano de uma mesma turma, numa escola pública de Lisboa, em que a primeira autora era também professora da turma. Esta situação implicou a tomada de medidas no sentido de minimizar a possibilidade de possíveis conflitos e enviesamentos. Assegurou-se a proteção dos alunos e das famílias, garantindo os princípios de honestidade, transparência e equidade no decurso de toda a investigação. Foi obtido o consentimento voluntário e informado de participação dos alunos e foram tomadas medidas para salvaguardar o seu anonimato e privacidade.

Os dados foram recolhidos a partir dos registos da observação participante, apoiados pelas gravações áudio e/ou vídeo das aulas, do diário de bordo da professora e das produções escritas dos alunos. A sua análise envolveu estratégias de indução analítica, a comparação constante, bem como a análise tipológica que permitiram definir categorias emergentes dos dados, atribuindo-lhes um significado ancorado no quadro conceptual que sustenta o estudo (Goetz \& LeCompte, 1984). Focamos a análise em seis tarefas selecionadas por permitirem destacar evidências categorizadas de compreensão dos aspetos conceptuais da percentagem 
(Lembke \& Reys, 1994, Moss \& Case, 1999; Parker \& Leinhardt, 1995), como: 1) usar conhecimento intuitivo para identificar a relação proporcional que traduz, apoiado em representações e na sua experiência do dia-a-dia; 2) usar conhecimentos dos números inteiros de 1 a 100, nomeadamente, estratégias de composição e decomposição, para interpretar o sentido de completude (sense of fullness) que oferece; 3 ) interpretar a relação de comparação de quantidades, considerando uma dada unidade; 4) usar valores de percentagem de referência, para estimar cálculos ou resolver problemas; e 5) tirar partido da sua relação com a representação decimal e com a fração, interrelacionando-as.

\section{Resultados}

\section{O sentido de completude da percentagem}

Começamos por analisar uma das tarefas iniciais da primeira etapa da trajetória. Nesta tarefa era pedido aos alunos que descobrissem que percentagem de um download poderia estar representada em duas barras de estado, uma representação icónica familiar aos alunos. Uma das estratégias espontâneas e intuitivas a que recorreram foi usar os dedos para medir e iterar, considerando que uma unidade é $100 \%$ (Figura 2).

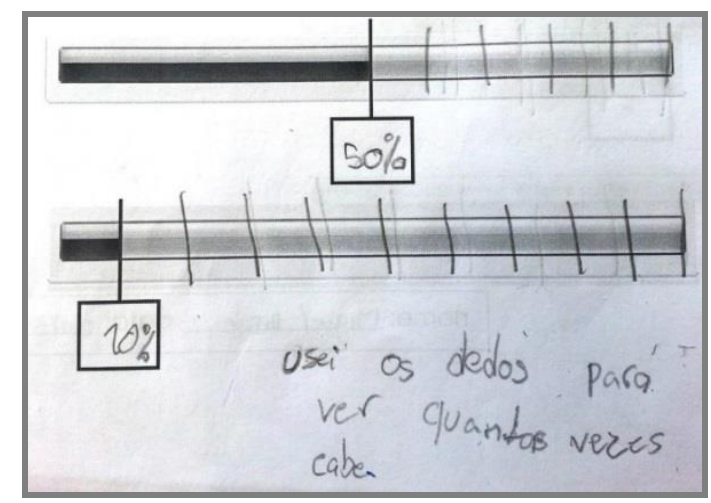

Figura 2 - Resolução da tarefa das barras de estado pelo grupo de Marco.

Fonte: Dados referentes a esta pesquisa.

Esta estratégia envolvia considerar à partida a barra de estado totalmente preenchida como o todo - $100 \%$ - e antecipar uma forma de determinar quantas vezes a parte considerada cabia nesse todo. A representação icónica foi usada como modelo da situação permitindo construir a estratégia de resolução. A representação simbólica emergiu como medida da distância correspondente à parte processada em cada uma das barras de estado, em relação ao tamanho da unidade, remetendo para um significado de medida.

Numa outra tarefa, foi pedido que os alunos estimassem, em percentagem, a quantidade de energia de uma bateria (Figura 3). 


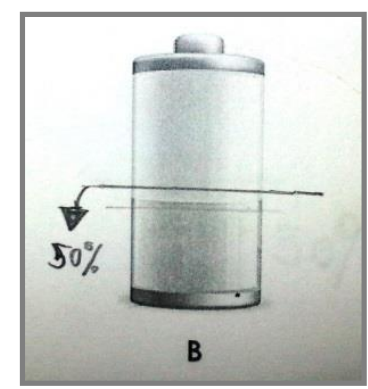

Figura 3 - Resolução da tarefa da bateria pelo grupo de Dinis.

Fonte: Dados referentes a esta pesquisa.

Alguns alunos sugeriram que estaria 50\% carregada, no entanto, outros, pela sua experiência, foram mais precisos na apreciação da quantidade de energia em relação ao tamanho da bateria quando completamente carregada:

Professora: Alguém acha que representa outra coisa?

Dinis: $\quad 40 \%$.

Professora: $\quad 40 \%$, porquê?

Dinis: $\quad$ Porque quando estou no tablet a jogar e vejo a bateria assim, sei que tenho mais ou menos $40 \%$.

Professora: Aparece lá escrito o valor em percentagem?

Dinis: $\quad$ Sim.

Professora: Mas aqui não tem nada escrito, por que é que não é 50\%?

Dinis: $\quad$ Porque teria que estar mais para cima, teria que estar a metade.

Embora os alunos evidenciassem um entendimento da compreensão de 50\%, as divergências surgiram em relação à precisão na medida da quantidade representada na imagem, como mostra a justificação do grupo de Ivo (Figura 4).

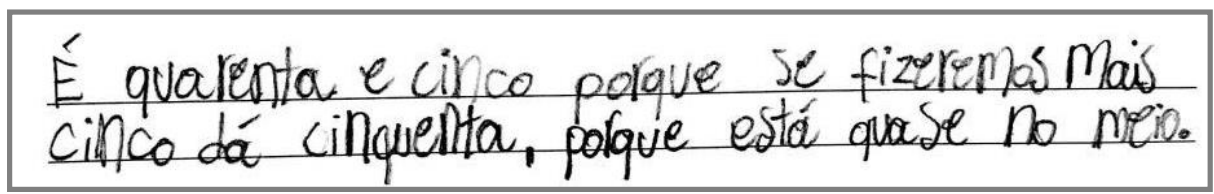

Figura 4 - Resposta do grupo de Ivo à tarefa da bateria.

Fonte: Dados referentes a esta pesquisa.

Na sua explicação, Ivo considerou que a bateria "está quase no meio", pelo que, para este aluno, seria possível que a quantidade representada fosse apenas $45 \%$, estimando um valor de 5\% em falta para chegar aos 50\%. A interação em torno desta justificação evidencia que os alunos mobilizaram relações aditivas para compreender que a consideração das diferentes partes em percentagem dava $100 \%$, apelando a um sentido de completude que a percentagem oferece, através de estratégias de composição e decomposição dos números inteiros de 0 a 100.

Nesta etapa, quando os alunos estão a avaliar um download ou estimar a quantidade de energia de uma bateria, a interpretação dada, embora se apoie na relação parte-todo, 
assume a parte num sentido de completude ao longo de uma escala linear, que varia entre $0 \%$ e $100 \%$ e que tem subjacente uma ideia de continuidade numérica, apoiada em conhecimentos dos números inteiros de 0 a 100. O valor em percentagem que os alunos atribuem à parte é percecionado como a comparação entre a medida que essa parte assume em relação ao todo (100\%), apoiada nas relações de proporção que a percentagem envolve.

\section{A percentagem como relação de comparação}

Avançando na experiência de ensino, convocamos para análise uma tarefa do fim da primeira etapa da trajetória e outra do início da segunda. A primeira tarefa surge a propósito de uma visita de estudo, em que a turma tinha escrito à instituição a solicitar um desconto. A resposta trouxe consigo um desconto de $3 €$ no preço do bilhete, cujo custo inicial era $12 €$. Esta situação foi aproveitada para investigar o valor do desconto em percentagem. A maioria dos grupos recorreu à reta numérica como base para o seu raciocínio. Sabendo o custo do bilhete antes do desconto e o valor, em euros, do desconto, o grupo de Ivo calculou o valor da percentagem correspondente verificando quantas vezes a parte cabia na unidade, recorrendo a um significado de medida (Figura 5).

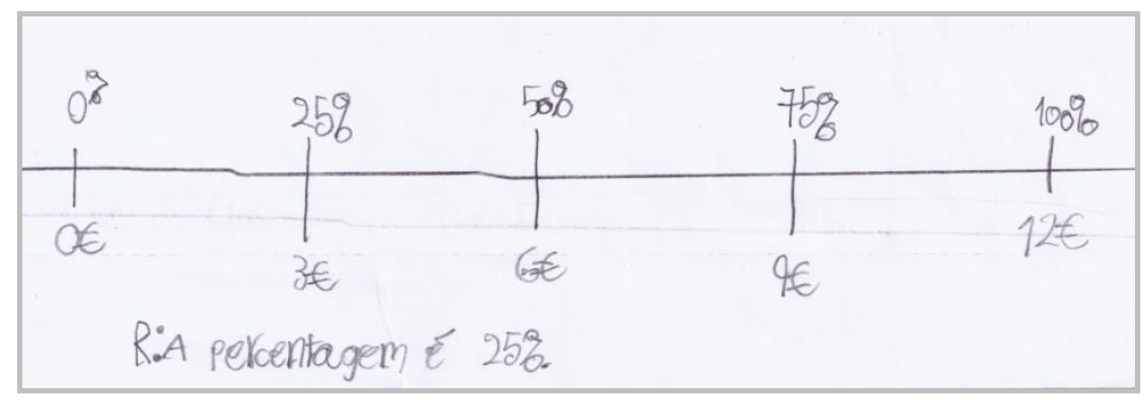

Figura 5 - Resolução do grupo de Ivo à tarefa através de uma reta numérica dupla.

Fonte: Dados referentes a esta pesquisa.

Ao identificarem os três euros do desconto, os alunos foram verificar quantas vezes essa quantia cabia no total e perceberam que conseguiam fazê-lo quatro vezes, recorrendo à divisão da unidade e iteração da parte. As marcações na reta emergiram assim deste raciocínio que foram construindo e que explicitaram no momento de discussão coletiva.

Professora: $\quad[\ldots]$ Que percentagem é de desconto? Martim

Martim: 25.

Dinis: $\quad 25 \%$.

Professora: Pois é! 25\% É o mesmo que dizer que me descontaram quanto?

Clara.

Clara: $\quad$ Metade da metade.

Ivo: $\quad$ Um quarto.

Professora: E porque é que é um quarto?

Ivo: $\quad$ Porque pagamos $75 \%$.

Com recurso à reta numérica dupla, os alunos convocaram a representação em fração, relacionando-a com a percentagem, alicerçada em conhecimentos numéricos anteriores 
procurando diferentes representações para o mesmo número, na tentativa de o tornar comum na sua explicação. A justificação apresentada por este grupo apoia-se no conhecimento de que $75 \%$ somado com um quarto, da mesma unidade, dá a própria unidade, mobilizando assim as duas representações simbólicas de forma complementar.

A segunda tarefa surgiu do facto de um aluno ter constatado, e partilhado com a turma, que num jogo de futebol, a equipa que teve maior tempo de posse de bola não foi a que ganhou o jogo. A propósito desta discussão foi construída esta tarefa, em que era apresentado um gráfico circular, retirado de um jornal diário, que mostrava a distribuição do tempo de posse de bola durante o jogo. Os alunos eram convidados a descobrir o tempo, em minutos, que cada equipa teria jogado, sabendo que a percentagem correspondente era de $45 \%$ para o Benfica e $55 \%$ para o Sporting. Trata-se de um contexto que fazia sentido para os alunos, pelo que, em interação, iam avaliando a razoabilidade dos cálculos e das suas interpretações:

Professora: Vamos lá ver, se uma equipa tiver $100 \%$ de posse de bola, quantos minutos são de posse de bola?

Heitor: $\quad 90$ minutos.

Simão: $\quad$ Mas isso é impossível!

Marco: $\quad$ Num jogo não pode haver uma equipa com $100 \%$ de posse de bola.

Professora: Claro que na prática seria uma coisa improvável...

A discussão em coletivo centrou-se no debate das estratégias que cada grupo encontrou para descobrir o tempo em minutos. Facilmente, a turma associou $50 \%$ a metade da duração do tempo de jogo, 45 minutos. Depois, usando a estratégia da composição e decomposição dos números inteiros de 1 a 100, descobriram as relações entre a percentagem e o tempo correspondente em minutos, como explicitou Luís no excerto de diálogo seguinte:

Luís: $\quad$ Eu e a Dina fizemos assim, como nós sabíamos que era 45\%, nós fomos buscar o $40 \%$, que dava 36 minutos. E depois fizemos mais o $10 \%$, mas vimos que não era $10 \%$, eram $5 \%$, fizemos metade do $10 \%$ para dar $5 \%$, dividimos o 9 ao meio e vimos que dava 4 minutos e meio.

Para calcular $45 \%$, os alunos foram decompor $45 \%$ em $40 \%$ mais $5 \%$. Como o valor correspondente a $40 \%$ era conhecido, faltava só descobrir o $5 \%$, que chegaram através do cálculo da metade de $10 \%$. A representação decimal da metade de nove surgiu de forma espontânea, sendo que a maioria dos alunos chegou a 4,5, apesar de não ter havido um trabalho formal com esta representação e que, por isso, alguns grupos justificaram como mostra a Figura 6. 


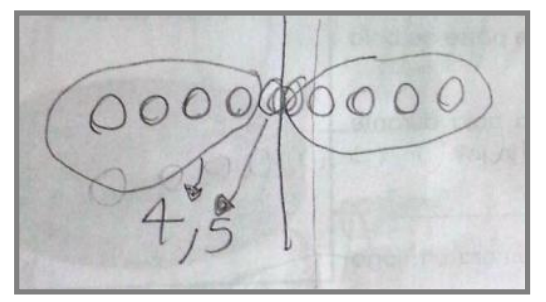

Figura 6 - Justificação do grupo de Horácio para o cálculo de metade de 9.

Fonte: Dados referentes a esta pesquisa.

Para descobrirem o tempo de posse de bola os alunos recorreram à estratégia de decomposição das percentagens, mobilizando as regularidades que conheciam. Em simultâneo, interpretaram a relação que estava associada à percentagem e encontraram diferentes formas de obter o valor em minutos correspondente a $55 \%$ de posse de bola, tal como mostra o registo da Figura 7.

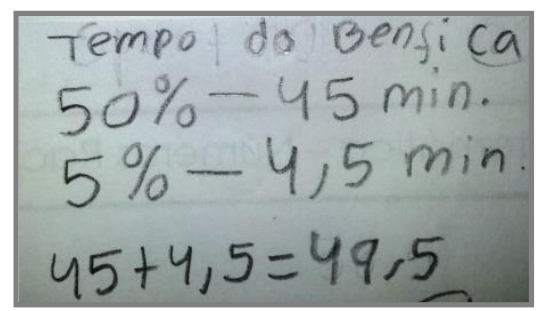

Figura 7 - Resposta do grupo de Bárbara para o tempo de posse de bola do Benfica.

Fonte: Dados referentes a esta pesquisa.

Esta tarefa apelava à compreensão das relações numéricas associadas à percentagem, num contexto significativo para os alunos. As estratégias de cálculo que os alunos construíram têm por base os conhecimentos prévios na sua relação com os novos números de referência $100 \%, 50 \%$ e 10\%, numa situação em que a partilha de tempo não é equitativa, pelo que os minutos relativos a cada equipa evidenciam uma distribuição proporcional.

Destacamos que os alunos conseguiram movimentar-se entre representações como a reta numérica dupla ou o gráfico circular, que se apoiaram num significado parte-todo de percentagem, e representações simbólicas, incluindo numerais decimais. Estabeleceram relações multiplicativas entre valores de conjuntos de natureza diferente: desconto em euros/percentagem do desconto e tempo de posse de bola/percentagem de posse de bola, interpretando a comparação entre quantidades específicas.

\section{A percentagem como proporção}

Reportamo-nos agora a uma tarefa da última etapa da trajetória de aprendizagem. Esta tarefa emerge da comunicação de um projeto sobre Planetas, apresentado à turma por um grupo de alunos. Nesta tarefa era pedido à turma que determinasse o diâmetro da Terra, tendo 
como referência o diâmetro de Júpiter, de aproximadamente 142000 quilómetros, e sabendo que o diâmetro da Terra seria aproximadamente $9 \%$ do de Júpiter.

A resolução da tarefa pelos diferentes grupos envolveu usar $10 \%$ e $1 \%$ como números de referência para o cálculo. Um dos grupos calculou $10 \%$ e depois $1 \%$, usando a divisão por 10, e fez a subtração (Figura 8).

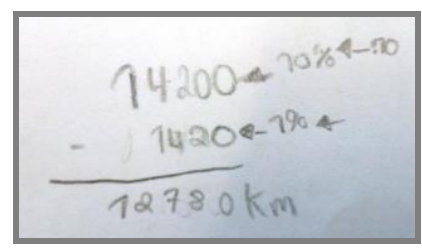

Figura 8 - Resolução da tarefa do diâmetro pelo grupo de Mafalda.

Fonte: Dados referentes a esta pesquisa.

Contudo, a maioria dos outros grupos, talvez devido à grandeza dos números envolvidos, mobilizou outros modelos para apoiar a construção da sua estratégia, nomeadamente, uma tabela de razão, como fez o grupo de Hélio (Figura 9).

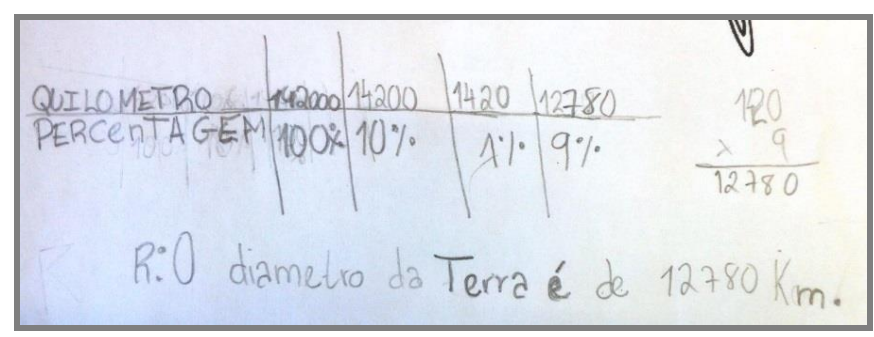

Figura 9 - Resolução da tarefa do diâmetro pelo grupo de Hélio.

Fonte: Dados referentes a esta pesquisa.

Este grupo recorreu ao cálculo de $10 \%$ e depois de $1 \%$, acabando por multiplicar $1 \%$ por nove. A tabela pareceu ter ajudado a estabelecer as etapas da estratégia seguida. O grupo de Clara também parece ter-se apoiado na reta numérica dupla, tendo registado as relações entre os valores em percentagem e os valores em quilómetros (Figura 10). Embora no início, tenham recorrido ao cálculo de outros valores de referência, como $50 \%$ e $25 \%$, acabam por chegar a $1 \%$ e usá-lo para determinar $9 \%$. 


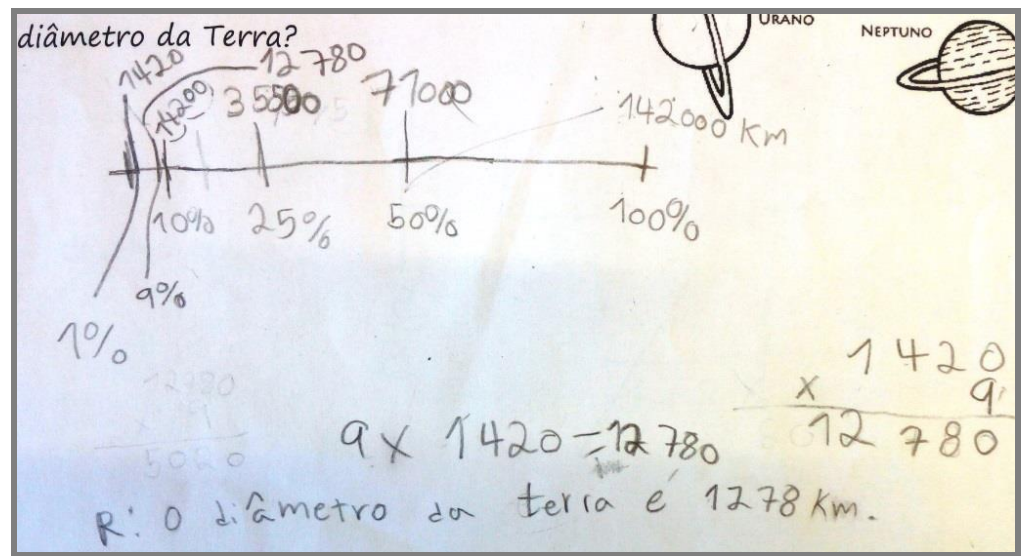

Figura 10 - Resolução da tarefa do diâmetro pelo grupo de Clara. Fonte: Dados referentes a esta pesquisa.

A reta numérica foi a estratégia mobilizada por outros grupos, como no caso do grupo de Ana (Figura 11). Este usou o modelo da reta numérica dupla para determinar o valor de $1 \%$, sem sentir necessidade de calcular $10 \%$, valor esse que multiplicou por nove, resolvendo assim o problema.

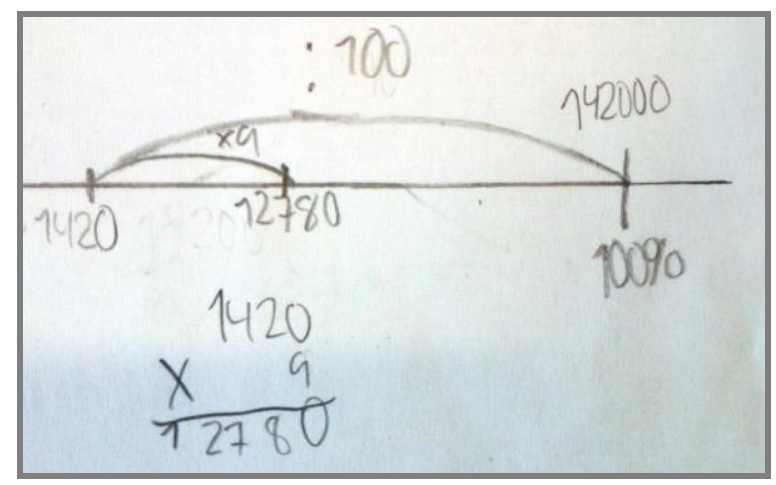

Figura 11 - Resolução da tarefa do diâmetro pelo grupo de Ana de Clara.

Fonte: Dados referentes a esta pesquisa.

Nesta tarefa, a percentagem foi interpretada como proporção, que os alunos mobilizaram apoiando-se em números de referência para fazer o cálculo. Este uso da percentagem sucedeu a um entendimento da sua dimensão relacional. As estratégias que os alunos construíram, mobilizando a construção de modelos relacionados com representações trabalhadas anteriormente, realçam a necessidade de verem explícitas as relações entre os referentes e as quantidades envolvidas, o que traduz uma compreensão da grandeza relativa da percentagem.

\section{A percentagem como representação de referência}

Por fim, analisamos uma das últimas tarefas da experiência de ensino, que teve lugar na terceira etapa da trajetória de aprendizagem. Esta tarefa envolvia a comparação de números representados em percentagem, fração e numeral decimal. Trata-se de uma tarefa de Zetetiké, Campinas, SP, v.26, n.2, mai./ago., 2018, 354-374 
contexto matemático, onde os alunos podiam usar como suporte uma reta numérica dupla, com diversos números de referência previamente marcados. O momento de discussão coletiva da tarefa procurou por em comum na turma algumas das estratégias usadas para identificar e comparar a grandeza dos números envolvidos, em diferentes representações, localizando-os sempre que possível e necessário na reta (Figura 12).

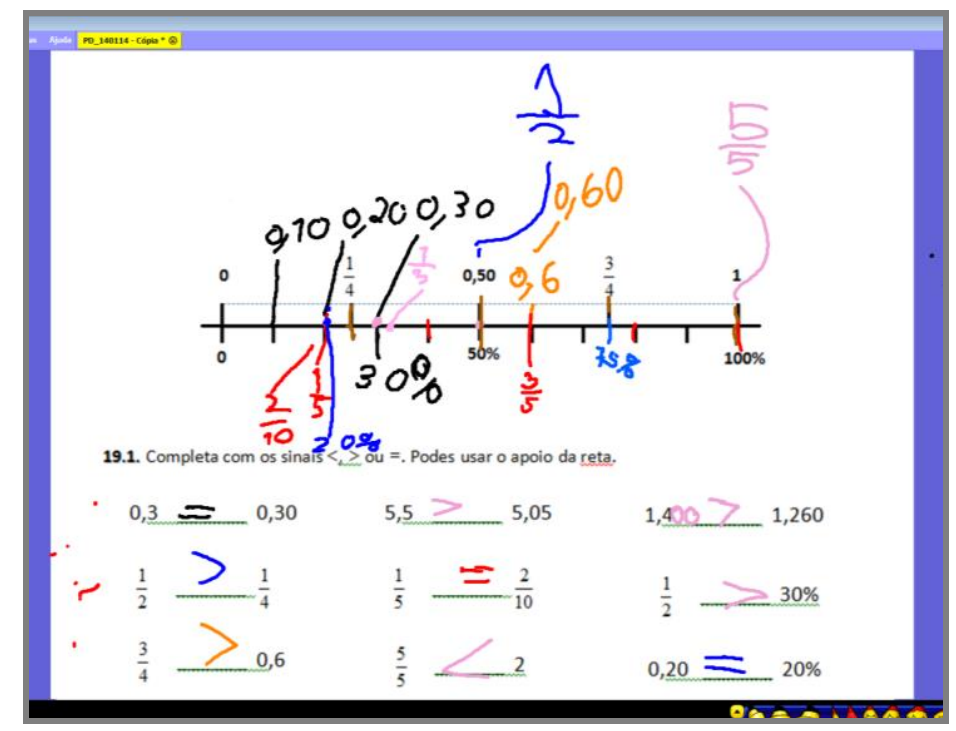

Figura 12 - Captura de écran do quadro interativo durante a discussão coletiva da tarefa.

Fonte: Dados referentes a esta pesquisa.

À medida que os alunos foram dando significado à grandeza dos números, a percentagem foi convocada para justificar as comparações que iam fazendo. Dado que 3/4 se encontrava marcado, Ana localizou na reta as seis décimas sem dificuldade e explicou a sua estratégia aos colegas:

Ana: $\quad$ Podemos fazer pedacinho a pedacinho. Como 50 centésimas é a mesma coisa que 5 décimas era só andar mais um e vemos que é o 6 décimas.

Professora: Mais um quê?

Ana: $\quad$ Mais um décimo.

Ana apoiou-se na reta, usando um significado de medida, para explicar à turma que as seis décimas correspondiam ao ponto que resultava de adicionar um décimo a cinco décimas ou seja cinquenta centésimas. De modo a envolver toda a turma na justificação da relação entre $3 / 4$ e 0,6 , os alunos foram convidados a identificar qual dos números representava um segmento de reta maior:

Professora: Olhem para reta. Vejam a posição do 3/4 e vejam as seis décimas. Qual é que representa um segmento de reta maior? Qual é que está mais perto do 1 ? Carolina.

Carolina: O 3/4.

Dinis: $\quad$ Eu vi que $3 / 4$ é $75 \%$ e que 6 décimas é $60 \%$, e assim vê-se logo... 
Um dos alunos da turma, Dinis, explicou que usou a percentagem para justificar esta relação, referindo que 3/4 representava o mesmo número que $75 \%$ e que 6 décimas representava o mesmo que $60 \%$, evidenciando fluência no uso de diferentes representações. A discussão avançou no sentido de partilhar outras estratégias:

Professora: Alguém pensou de outra forma?

Simão: $\quad$ Eu fiz o do $1 / 5$ e dividi em cinco e deu 3/5 nas 6 décimas e 3/5 é menos que $3 / 4$.

Professora: O Simão dividiu em quintos. Vem cá marcar.

$\cdots$

Simão:

O 3/5 é mais pequeno do que o 3/4 (apontando na reta).

Professora:

É outra forma de explicar. Assim, se pensarmos em dividir em 5 partes vemos que são divisões mais pequenas do que se dividimos em quatro. E, em percentagem, diz lá Dinis, o 3/4 é...

Dinis: $75 \%$.

Professora: E o 3/5? Vejam (aponta na reta)...

Humberto: $\quad$ São 6 décimas.

Márcia: $\quad 60 \%$.

Professora: $\quad 60 \%$, e então qual é que representa uma distância maior?

Alunos: $\quad 3 / 4$.

Heitor: $\quad$ É como na piza!

Professora: Isso, então 6 décimas (aponta na reta), que são os 3/5, é mais pequeno que $3 / 4$.

Neste excerto, o contributo da percentagem traduz-se na forma como esta permite interpretar a grandeza do mesmo número em diferentes representações. Usada como representação de referência, contribuiu para que os alunos compreendessem as frações $3 / 5$ e $3 / 4$, como correspondentes a $60 \%$ e $75 \%$. Deste modo, os alunos interpretaram corretamente as frações, sem fazerem associações inadequadas ou raciocínios abusivos a partir da sua experiência com os números inteiros.

\section{Discussão}

Pela sua utilização no dia-a-dia dos alunos e pela relação de base 100 que oferece, a percentagem ganha sentido à medida que permite "ligar os dois mundos" (Parker \& Leinhardt, 1995, p. 422), isto é, permite que a vida dê sentido à Matemática da escola e que esta seja interpretada como instrumento de compreensão da própria vida. Deste modo, numa etapa inicial da aprendizagem dos números racionais, procurámos perceber que compreensão os alunos constroem da natureza relacional da percentagem e de que modo a percentagem contribui para essa aprendizagem, considerando uma compreensão das relações entre as diferentes representações destes números.

$\mathrm{Na}$ experiência de ensino desenvolvida neste estudo, o trabalho realizado com a percentagem centrou-se na sua dimensão relacional tendo-se focado numa compreensão gradual dos aspetos conceptuais da percentagem (Moss \& Case, 1999; Parker \& Leinhardt, 1995). Inicialmente, remeteu para a construção de um sentido de completude, a partir do 
olhar dos alunos sobre representações associadas à percentagem dos contextos do dia-a-dia. Envolveu também a construção de estratégias de resolução espontâneas, que tinham por base os conhecimentos relativos aos números inteiros de 0 a 100, associados a uma compreensão intuitiva da natureza proporcional do sentido de completude oferecido pela percentagem. Esta etapa foi um marco importante no processo de alargamento do conhecimento aos números racionais, uma vez que os alunos compreenderam que uma percentagem representada pelo mesmo número pode traduzir quantidades diferentes, que correspondem a um todo também diferente, como uma barra de estado ou uma bateria, mas que se considera $100 \%$. Seguidamente, o trabalho realizado procurou promover a compreensão da noção de comparação multiplicativa expressa pela percentagem. Os alunos deram significado à percentagem enquanto relação entre dois números, duas quantidades de natureza diferente, como desconto em percentagem e valor em euros ou percentagem de tempo de posse de bola e minutos de posse de bola. Representações como a reta numérica dupla foram usadas como modelos para interpretar situações da realidade, com significado para os alunos, que se evidencia também na sensibilidade que mostram para apreciar a razoabilidade dos resultados. Posteriormente, tendo por base a ideia que a percentagem traduz uma proporção, desenvolveram-se tarefas tendo em vista a compreensão de que a percentagem está sempre relacionada com algo, o todo a que diz respeito. Os alunos mobilizaram números de referência, como $10 \%$ ou $1 \%$ para determinar uma quantidade proporcional em relação ao todo que se considera, numa proporção para 100, evidenciando um entendimento das relações multiplicativas associadas à noção de percentagem. A construção deste conhecimento conceptual por parte dos alunos evidencia uma compreensão emergente da natureza relacional da percentagem, enquanto noção com propriedades de número racional, que se desenvolveu progressivamente à medida que estabeleceram comparações multiplicativas de quantidades, compreendendo as relações envolvidas e coordenando unidades de referência.

Uma aprendizagem com compreensão dos números racionais envolve também, como relembram Tian e Siegler (2017), ser capaz de se movimentar entre as três representações simbólicas e saber escolher e usar a mais adequada a cada situação. Esta capacidade de entrelaçamento de diferentes representações na interpretação da grandeza de um número racional é evidenciada quando os alunos comparam números na primeira tarefa apresentada e descobrem, por exemplo, que 3/4 representa um número maior que 0,6 e também um número maior do que 3/5. Na verdade, estas descobertas aconteceram apoiadas em conversões que convocam a percentagem, com o apoio da reta numérica dupla, traduzindo a capacidade dos alunos se movimentarem entre representações. Estas conversões têm no seu âmago a compreensão da grandeza do número racional por elas representado, para a qual parecem ter contribuído as oportunidades criadas pelo trabalho com a percentagem. As diferentes situações que envolveram a percentagem permitiram aos alunos raciocinar proporcionalmente com quantidades, estabelecendo comparações e construindo um entendimento da natureza multiplicativa dos números racionais. 


\section{Conclusão}

Parker e Leinhardt (1995) e Moss e Case (1999) consideram que é nos níveis de escolaridade básica que devem ser lançadas as sementes para reconhecer a importância de 100 como base privilegiada e para sensibilizar para as relações comparativas que a percentagem oferece, maximizando a conexão com os conhecimentos originais e intuitivos de razão. Por um lado, centrada na compreensão das suas caraterísticas conceptuais, a percentagem constitui uma forma de dar significado à relação proporcional que oferece, integrando os conhecimentos relativos aos números inteiros. E, por outro lado, permite alicerçar um entendimento da natureza multiplicativa dos números racionais, na medida em que conduz a interpretar de forma intuitiva a relação de comparação que estes números traduzem, isto é, como afirmam Galen et al. (2008) a perceção da razão entre o que está a ser descrito e a unidade a que se refere. Nesta perspetiva, este estudo mostra que a percentagem constitui conteúdo relevante e adequado para ser trabalhado nesta etapa da escolaridade, permitindo aprendizagens consistentes por parte dos alunos. Muito especialmente, a percentagem destaca-se como uma representação de referência num processo integrado de extensão dos conhecimentos dos números inteiros aos números racionais e contribui para uma aprendizagem dos números racionais com compreensão, apoiada na inter-relação entre representações.

\section{Agradecimentos}

Este trabalho é financiado por fundos nacionais através da Universidade de Lisboa, no âmbito do Programa de Bolsas de Doutoramento, através de uma bolsa atribuída à 1. a autora.

\section{Referências}

Behr, M., Lesh, R., Post, T., \& Silver, E. (1983). Rational number concepts. In R. Lesh \& M. Landau (Eds.), Acquisition of mathematics concepts and processes (pp. 91-126). New York, NY: Academic Press.

Behr, M. J., Lesh, R., \& Post, T. (1981, April). Rational number ideas and the role of representational systems. Paper presented at the Annual Meeting of the American Educational Research Association, Los Angeles, USA.

Bruner, J. (1996). Cultura da educação. Lisboa: Edições 70.

Cobb, P., Stephan, M., McClain, K., \& Gravemeijer, K. (2001). Participating in classroom mathematical practices. In E. Yackel, K. Gravemeijer \& A. Sfard (Eds.), A journey in mathematics education research: Insights from the work of Paul Cobb (pp. 117-163). New York, NY: Springer.

De Corte, E., Depaepe, F., Op’t Eynde, P., \& Verschaffel, L. (2005, January). Comparing mathematics education traditions in four European countries: The case of the teaching of percentages in the primary school. Paper presented at the meeting of Universiti Teknologi Malaysia, Malaysia. 
Dreyfus, T. (1999). Why Johnny can't prove. In D. Tirosh (Ed.), Forms of mathematical knowledge (pp. 85-109). Dordrecht: Springer.

Freudenthal, H. (1968). Why to teach mathematics so as to be useful. Educational Studies in Mathematics, 1(1), 3-8.

Goetz, J. P., \& LeCompte, M. D. (1984). Ethnography and qualitative design in educational research. New York, NY: Academic Press.

Goldin, G. A., \& Kaput, J. J. (1996). A joint perspective on the idea of representation in learning and doing mathematics. In L. Steffe, P. Nesher, P. Cobb, G. A. Goldin, \& B. Greer (Eds.), Theories of mathematical learning (pp. 397-430). Hillsdale, NJ: Eribaum.

Gravemeijer, K., \& Cobb, P. (2006). Design research from the learning design perspective. In van den Akker, K. Gravemeijer, S. McKenney, \& N. Nieveen (Eds.), Educational Design Research (pp. 45-85). London: Routledge.

Hiebert, J., \& Lefevre, P. (1986). Conceptual and procedural knowledge in mathematics: An introductory analysis. In J. Hiebert (Ed.), Conceptual and procedural knowledge: The case of mathematics (pp. 1-27). Hillsdale, NJ: Erlbaum.

Hiebert, J., Wearne, D., \& Taber, S. (1991). Fourth graders' gradual construction of decimal fractions during instruction using different physical representations. The Elementary School Journal, 91(4), 321-341.

Lamon, S. (2007) Rational numbers and proportional reasoning. In F. Lester (Ed), Second handbook of mathematics teaching and learning (pp. 629-667). Greenwich, CT: Information Age.

Leinhardt, G. (1988). Getting to know: Tracing students' mathematical knowledge from intuition to competence. Educational Psychologist, 23(2), 119-144.

Lembke, L. O, \& Reys, B. J. (1994). The development of, and interaction between, intuitive and school-taught ideas about percent. Journal for Research in Mathematics Education, 25(3), 237-259.

Moss, J. (2002). Percents and proportion at the center: Altering the teaching sequence for rational number. In B. Littweiller (Ed.), Making sense of fractions, ratios, and proportions (pp. 109-120). Reston, VA: National Council of Teachers of Mathematics.

Moss, J., \& Case, R. (1999). Developing children's understanding of the rational numbers: A new model and an experimental curriculum. Journal for Research in Mathematics Education, 30(2), 122-147.

NCTM (2000). Principles and standards for school mathematics. Reston, VA: National Council of Teachers of Mathematics.

Parker, M. (2004). Reasoning and working proportionally with percent. Mathematics Teaching in the Middle School, 9(6), 326-330.

Parker, M., \& Leinhardt, G. (1995). Percent: A privileged proportion. Review of Educational Research, 65(4), 421-481.

Ponte, J. P. (2005). Gestão Curricular em Matemática. In GTI (Ed..), O professor e o desenvolvimento curricular (pp.11-34). Lisboa: APM. 
Ponte, J. P., Carvalho, R., Mata-Pereira, J., \& Quaresma, M. (2016). Investigação baseada em design para compreender e melhorar as práticas educativas. Quadrante, 25(2), 77-98.

Ponte, J. P., \& Serrazina, M. L. (2000). Didáctica da Matemática do $1 .^{\circ}$ ciclo. Lisboa: Universidade Aberta.

Serrazina, M. L. (2012). O sentido do número no $1^{\circ}$ ciclo: Uma leitura de investigação. Boletim Gepem, 61, 15-28.

Siegler, R. S. (2016). Magnitude knowledge: The common core of numerical development. Developmental Science, 19(3), 341-361.

Siegler, R. S., Fazio, L. K., Bailey, D. H., \& Zhou, X. (2013). Fractions: The new frontier for theories of numerical development. Trends in Cognitive Sciences, 17(1), 13-19.

Siegler, R. S., Thompson, C. A., \& Schneider, M. (2011). An integrated theory of whole number and fractions development. Cognitive Psychology, 62(4), 273-296.

Tian, J., \& Siegler, R. S. (2017). Which type of rational numbers should students learn first?. Educational Psychology Review, 1-22.

Van den Heuvel-Panhuizen, M. (2003). The didactical use of models in realistic mathematics education: An example from a longitudinal trajectory on percentage. Educational Studies in Mathematics, 54(1), 9-35.

Van Galen, F., Feijs, E., Figueiredo, N., Gravemeijer, K., Herpen, E., \& Keijzer, R. (2008). Fractions, percentages, decimals and proportions: A learning-teaching trajectory for grade 4, 5 and 6. Rotterdam: Sense.

Van Galen, F., \& Van Eerde, D. (2013). Solving problems with the percentage bar. Indonesian Mathematical Society Journal on Mathematics Education, 4(1), 1-8. 